\title{
Mission Concepts Utilizing a Laser Communications and DTN-Based GEO Relay Architecture
}

\author{
David J. Israel \\ NASA/GSFC Code 450 \\ Greenbelt, MD 20771 \\ 301-286-5294 \\ dave.israel@nasa.gov
}

\author{
Bernard L. Edwards \\ NASA/GSFC Code 560 \\ Greenbelt, MD 20771 \\ 301-286-8926 \\ bernard.l.edwards@nasa.gov
}

\author{
Donald E. Whiteman \\ NASA/GSFC Code 599 \\ Greenbelt, MD 20771 \\ 301-286-2327 \\ donald.e.whiteman@nasa.gov
}

\begin{abstract}
Laser Communications and Delay Tolerant Networking (DTN) are two key technologies in development for enhancement of future generation space communications. Laser Communications or lasercom holds the potential for orders of magnitude increases in data rates while also reducing communications system size, weight, and power. DTN will allow for networked communications across space links maximizing network capacity and scalability. These two technologies combined on a geosynchronous Earth relay satellite will enable new and enhanced mission concepts. The Laser Communications Relay Demonstration (LCRD) project, scheduled for launch in December 2017, will demonstrate these technologies and address the remaining challenges for the new architecture. This paper describes mission concepts that will be demonstrated as part of LCRD with an emphasis on how lasercom and DTN will enable and enhance future mission operations and data return.
\end{abstract}

\section{Table of Contents}

1. INTRODUCTION .1

2. LASER COMMUNICATIONS ..............................2

3. DTN/NETWORKING.....................................4

4. SUMMARY/CONCLUSION ...............................5

REFERENCES.....................................................6

\section{INTRODUCTION}

The LCRD mission is a Principal Investigator led project funded by the NASA Office of Chief Technologist (OCT) and the Space Communications and Navigation ( $\mathrm{SCaN}$ ) program. Its goal is to demonstrate that laser communications can meet the ever-increasing need to implement high rate communications using low power, low mass laser communications systems on board future spacecraft. The LCRD mission will develop and deliver onorbit all of the critical hardware and software technologies that will be needed to support a communications architecture required by future space missions that are expected to transmit and receive very high data rates and very large volumes of earth- and space-science data. A key aspect of this future communications architecture will be the ability to relay data from one spacecraft to another via wide bandwidth optical links. The technologies that need to be demonstrated include, but are not necessarily limited to, the following: optical components and electronics used to implement an integrated DPSK/PPM modem, optical system pointing and tracking, and networking protocols. The LCRD project will use mature, proven, existing systems U.S. Government work not protected by U.S. copyright whenever possible, and it will leverage existing flight system technologies by implementing modifications and enhancements as needed to fully characterize high data rate laser communications in a space flight environment. LCRD will provide two years of continuous high data rate laser communications in an operational environment.

The high data rates possible over optical links are expected to reduce life-cycle costs for mission operations and infrastructure by enabling large data volumes to be directly downlinked to fewer, more accessible locations, in contrast to the current RF based space and ground communications network. The dual optical link system also enables LCRD to become the first step in demonstrating laser communications for the next generation Tracking and Data Relay Satellite (TDRS), and it has the potential to provide early operational support for proposed International Space Station (ISS) and other low Earth orbit (LEO) terminals. The LCRD mission advances laser communications technology toward infusion into Deep Space and Near Earth operational systems, while growing the capabilities of industry sources.

The LCRD mission architecture also has the potential to demonstrate the space-based networking relay operations. The demodulation and remodulation within the flight payload allow for the onboard operation of higher layer protocols, such as the Delay Tolerant Networking (DTN) Bundle Protocol. Couple with systems within the LCRD ground segment, a full instantiation of a space internetworking service provider can be demonstrated.

LCRD is comprised of space and ground based elements as illustrated in Figure 1. The LCRD mission will leverage existing JPL and MIT LL assets to provide ground station optical terminal support at Table Mountain, CA and White Sands. NM. These locations are supporting the Lunar Laser Communications Demonstration (LLCD) mission and LCRD components will be incorporated into the existing ground station systems to allow them to support LCRD experiment. The LCRD geosynchronous (GEO) flight payload including optical terminals will be hosted onboard a Space Systems Loral communications satellite. Payload commands and telemetry will be routed through an RF communications link to the Host spacecraft operator's mission operations center (HMOC). The LCRD mission operations center (LMOC) located at NASA GSFC coordinates ground station and payload operations. 


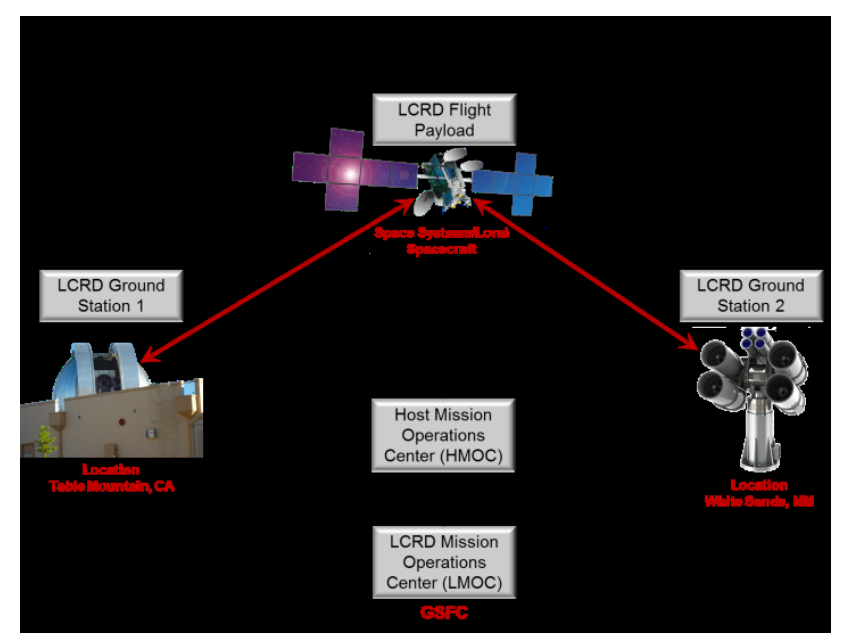

Figure 1 LCRD Mission Architecture

\section{LASER COMMUNICATIONS}

LCRD provides two years of continuous high data rate laser communications in an operational environment. High-rate communications will revolutionize science and exploration. Laser communications has the potential to provide for data rates 10-100 times more capable than current RF systems, allowing new kinds of connectivity paradigms and enabling a new generation of remote scientific and planetary investigations. Laser communications will enable missions to use bandwidth-hungry instruments, such as hyperspectral imagers, synthetic aperture radar (SAR), and instruments with high definition in spectral, spatial, or temporal modes. Laser communications will make it possible to establish a "virtual presence" at a remote planet or other solar system body, enabling high-rate communications with future explorers.

LCRD with utilize an Integrated Modem that provides for both Differential Phase Shift Keying (DPSK) and Pulse Position Modulation (PPM) to be used in the demonstration. The modulation type utilized for each experiment will be based on required performance characteristics and applicability to support specific mission scenarios being simulated. The Integrated Modem modulates the input electrical data into an optical signal for transmission and demodulates a received optical signal into output electrical data. The modulation, waveforms and physical layer framing used on the optical signals are designed to enable high-sensitivity, adjustable data-rate, bi-directional optical communication over a fading, free-space communication channel.

The first modulation type, Pulse Position Modulation (PPM) is highly efficient and the modulation of choice for Deep Space communications or constrained (very low power/mass systems) near-earth links when implemented with photon counting detectors. M-ary PPM uses an on-off keying approach to map data bits into slots within a frame and this technique offers greater efficiency for high peak to average power ratio operations required for power constrained links. This is due to the resulting operation of the optical amplifier at a lower duty cycle. The use of PPM along with photon counting techniques in the detector increases the receive system's sensitivity and enables enhanced communications performance for deep space missions. However, LCRD only has a photon counting detector in one of the ground stations due to budget constraints. The ultimate data rate is limited based on detector limitations and the requirement for faster electronics due to internal clock rates needed to support higher data rates. This modulation type is limited to maximum User data rates up to $311 \mathrm{Mbps}$ for LCRD. User data are defined as data available for network users and exclude overhead related to frame headers, coding and interleaving.

The flight payload can support communications when the sun is in the field of view (FOV). This solar advantage is derived from both coupling into a single mode fiber (spatial mode selectivity) and from optical filtering (spectral selectivity). On the flight terminal there will be no photon counters so both received PPM and DPSK will be coupled into single mode fiber and spectrally filtered. This provides a solar advantage if it is necessary to point near the limb of the earth with the sun behind or when staring near bright solar scatter/reflections.

The basic laser communications concept of operations assumes there is a specific amount of data at the source that must be transferred to the destination in a specific amount of time - not unlike a typical RF scenario. The high-level process assumes a scheduled approach where the communications process starts at a specific time, with a link establishment process between the source spacecraft and the destination (ground station or relay satellite). If the link is successfully established, data is transferred during the specified time and then the link is terminated according to plan. If link establishment is not successful or the link cannot be maintained for the required duration, due to clouds or other link impairment, then an alternative process is required. At a minimum, the data not transferred must be stored until it can be transferred later (to another ground station or relay) or deleted.

The first consideration in link establishment is whether a line of sight between the source and destination exists. Geometric line of sight is calculated based upon the source spacecraft trajectory, location of the ground station(s), and any local terrain considerations, e.g., mountains, buildings, trees, etc. Free space laser communications through Earth's atmosphere is nearly impossible in the presence of most types of clouds. Typical clouds have deep optical fades and therefore it is not feasible to include enough margin in the link budget to prevent a link outage. Thus a key parameter when analyzing free space laser communications through the atmosphere is the probability of a cloud-free line of sight (CFLOS) channel. Therefore, a mitigation technique ensuring a high likelihood of a CFLOS between the source and destination is needed to maximize the transfer of data 
and overall availability of the network. Such mitigation techniques include:

- Using several laser communications terminals on the relay spacecraft, each with its own dedicated ground station, to simultaneously transmit the same data to multiple locations on Earth; hopefully one will be free of clouds to allow the transmission to succeed. Unfortunately, this mitigation technique can be cost prohibitive.

- A single laser communications terminal in space can utilize multiple ground stations that are geographically diverse, such that there is a high probability of CFLOS to a ground station from the spacecraft at any given point in time.

- $\quad$ Storing data until communications with a ground station can be initiated. Of course a key issue is making sure all of the stored onboard data can be transmitted within the allocated time.

- Having a dual RF / laser communications systems onboard the spacecraft. The RF system could support low data rate low latency communications while the laser communications could support high data rate communications of data that can be stored for some time

NASA has studied various concepts and architecture for a future laser communications network. The analysis indicates ground segment solutions are possible for all scenarios, but usually require multiple, geographically diverse ground stations in view of the spacecraft. Thus laser communications handover strategies will have to be developed. Those strategies may be different depending upon the transmission time required; e.g. handover strategies that work with a laser communications terminal in Low Earth Orbit may not work for a laser communications terminal located at Mars.

The availability of a communication link between a spacecraft and a ground station network depends on many factors, including the number and location of the sites in the network and the orbit of the spacecraft, which together determine the elevation angle of the link and the path length of transmission through the atmosphere. In our concept, a ground station is considered "available" for communication when it has a CFLOS at an elevation angle to the spacecraft terminal of approximately $20^{\circ}$ or more. The network is "available" for communication when at least one of its sites is "available." Typical meteorological patterns cause the cloud cover at stations within a few hundred kilometers of each other to be correlated. Consequently, stations within the network should be placed far enough apart to minimize these correlations, thus maximizing the probability of CFLOS. This requirement may lead to the selection of a station that has a lower CFLOS than sites not selected, but is less correlated with other network sites. The stations also need to be close enough to each other to maintain continuous access with the spacecraft as its position with respect to the ground changes with time.

Depending on the scenario, free space laser communications operations can take advantage of cloud prediction at each ground site to maintain CFLOS, and thus maximize availability. Maintenance of CFLOS can be accomplished by knowing whether the line of sight to each ground site is cloud free at a given time, and knowing how many minutes into the future each site is expected to remain cloud free. Having local weather and atmospheric instrumentation at each site and making a simple cloud forecast can significantly reduce the amount of time the space laser communications terminal requires to re-point and acquire with a new ground station. Useful instrumentation includes:

- Weather: Weather information is gathered locally by standard meteorological packages that monitor temperature, humidity, barometric pressure, and wind speed and direction.

- Clouds: A thermal infrared cloud camera is used to monitor the extent of cloud coverage. These sensors indicate not only whether there are clouds or no clouds at very high temporal resolution, but also the sky temperature and emission.

- Daytime Sky Radiance: A sun photometer provides this measurement.

- Atmospheric Loss: During the day, a sun photometer is also used to measure atmospheric loss. At night, a calibrated photometric system that tracks stars of stable emission, e.g., Polaris, can be used.

- Clear Air Optical Turbulence: A Differential Image Motion Monitor (DIMM) is the predominant method of measuring seeing. During the night this instrument tracks stars and during the day, the Sun.

In addition to outages or blockages due to weather, a laser communications link also has to be safe. Besides needing to be safe for humans, some satellites have sensitive detectors that can be damaged by intense light sources originating from Earth. Safe laser beam propagation to near-Earth and deep space spacecraft starts at the transmitter, extends through navigable air space and the near-Earth region, and eventually into deep space. In the United States, regions of concern for safe beam propagation are under the purview of the Occupational Safety and Health Administration, (OSHA) the Federal Aviation Administration (FAA) and the Laser Clearing House (LCH), respectively.

As a federal government agency, NASA generally coordinates its outdoor laser activities with the Laser Clearinghouse, a Department of Defense component within the United States Strategic Command. The ANSI standard for outdoor laser propagation (ANSI Z136.6) also 
recommends that lasers that meet certain criteria also coordinate with the Laser Clearinghouse.

The Laser Clearinghouse provides predictive avoidance analysis and deconfliction with US, allies, and other space operations due to the potential to affect satellites or humans in space. The Laser Clearinghouse can determine whether a laser has the potential for interference or damage to particular satellites. Lasers with very high peak powers and very small beam divergences (10 microradians or less) probably have the greatest potential for producing damage to these very expensive systems in space. Rather than give detailed guidance on the levels that could produce damage to satellites, criteria are provided as a starting place where coordination with the Laser Clearinghouse is desirable. Lasers that can produce an instantaneous irradiance exceeding $1 \mathrm{~mW} \mathrm{cm-2}$ at an elevation of 60,000 feet (18 $\mathrm{km}$ ), even for $1 \mathrm{~ns}$ or less, should contact the Laser Clearinghouse. Based on the laser propagation details, the Laser Clearinghouse can either issue a blanket approval of transmission at a facility or require coordination of all laser beam propagation activity.

Thus a laser safety system and operational procedures have to be in place to ensure safe operations. Those procedures may lead to periodic outages of the link just as if a cloud had blocked a station.

As laser communication links through the atmosphere will be intermittently interrupted, the use of data return acknowledgement and retransmission protocols, such as Delay Tolerant Networking (DTN), help improve the data throughput and completeness. Such protocols cope with atmospheric disturbances to the laser communications link and additionally prevent the loss of data during the redirection of the downlink to a second ground station if the first should be obscured by clouds. Data acknowledgment requires sending information back to the spacecraft either through an optical uplink, or alternatively through an RF uplink. Since the data rates on optical communication links will typically be higher than those of RF links, research should be conducted on the best data return acknowledgement and retransmission protocols for use with optical links.

Adaptive optics (AO) will be utilized to improve the performance of the LCRD optical system by reducing wavefront distortion effects. These include atmospheric perturbations due to turbulence. The adaptive optics compensates for optical aberrations caused by atmospheric turbulence along the propagation path. AO has been commonly used since the 1990's in astronomy and more recently in laser communication systems to mitigate wavefront distortion effects. The LCRD system will also operate at a higher bandwidth and lower elevation angles than existing systems.

\section{DTN/NETWORKING}

The onboard processing in the LCRD payload differs from the current TDRS "bent-pipe" system, enabling new relay services. The LCRD architecture allows the project to serve as a testbed for the development and demonstration of network layer relay services. These developments and demonstrations will apply to optical, non-optical, and hybrid future communication systems.

The first challenge for providing the higher layer data services is the inclusion of onboard data processing. The onboard system must be able to perform demodulation, decoding, de-interleaving, and link layer frame processing, in order to perform functions based on the payload data stream and structures. This processing requires high speed hardware designs since it must happen at the received symbol and frame rates. The maximum rates that can be supported by these onboard processes have typically been the limiting factor for the maximum data rates available for higher layer services. The complementary functions must also be performed in the transmit direction, however those functions are generally less complex and less constraining. The LCRD requirements for this capability are for data rates up to $300 \mathrm{Mbps}$.

The higher layer services to be provided by LCRD include link layer and network layer services. Link layer services will be based on the use of CCSDS AOS frame structures. The fundamental link layer service provided will be the capability to use virtual channels to separate data streams at the frame level. This capability will allow onboard separation of different types of data and different user data streams.

Network layer services allow the use of routing protocols to automatically forward data onto the next link based on information within received data stream. LCRD will implement network layer services based on the DTN Bundle Protocol (BP). In a DTN, asynchronous variable-length messages (called bundles) are routed in a store and forward manner between participating nodes over a heterogeneous network. The use of DTN enables autonomous store and forward operations, by providing network layer services and storage at intermediate nodes.

For the GEO relay service provider, the addition of networking and, specifically, DTN allows increase operational flexibility and efficiencies with increased complexity. Through the use of multiplexing user data streams at a combination of link layer and network layer multiplexing, a single space-to-ground link or trunkline between the relay spacecraft and Earth can carry all user traffic. Scheduled or autonomous routing onboard can forward data over the appropriate link to the destination. The appropriate link can also include a crosslink to another GEO relay.

Through the use of onboard storage, trunkline data can be transferred at rates other than the actual user forward and 
return link data rates. The trunkline data can even include data for links not currently available, either due to planned or unplanned link outages. These capabilities are especially critical for optical link relays to allow for disruptions in the optical space-to-ground link.

For the relay provider, however, these capabilities require additional relay and ground functionality and increased operations complexity. Onboard storage is required on the relay. The amount of storage, the speed at which the storage can be accessed, and the number of simultaneous links that can access the storage are all performance parameters that can limit the functionality of the relay system. LCRD is currently carrying requirements to support two simultaneous bi-directional links at up to $1.25 \mathrm{Gbps}$ with ten minutes of storage. The relay payload must also implement the link layer and network layer protocols. The complexity of these protocols, combined with the desired data rates, will likely require a combined hardware and software design. The storage and protocol support is also required at the relay provider's ground stations. A networking management system must be in place, as well, that will provide the relay provider with the full insight required to ensure user data delivery and enough control to manage the schedule for all links and routing updates.

The LCRD payload, ground stations and mission operations center (MOC) should have the capability to implement and test a variety of DTN techniques during the planned experimental scenarios. These include published bundle protocol, transport protocol, and transfer acknowledgement methods, as well as the flexibility to support some additional yet-to-be determined DTN techniques through software or firmware changes to the LCRD spacecraft and ground based systems.

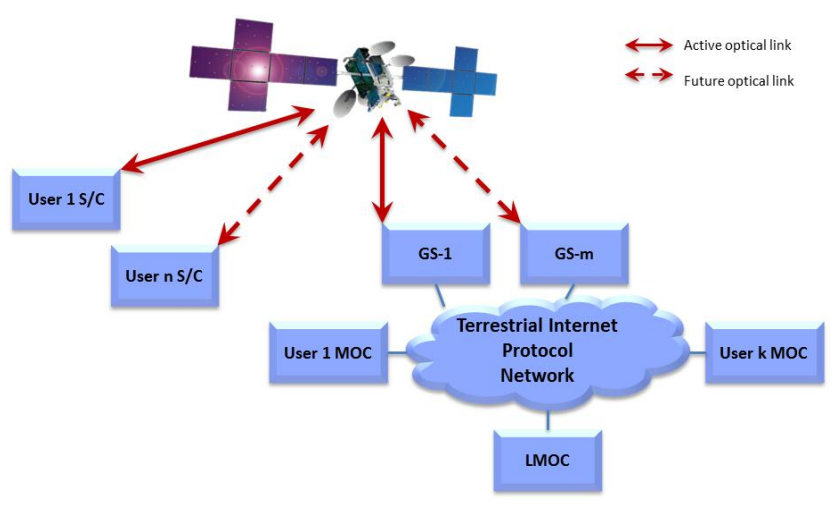

Figure 2 LCRD Design Reference Mission Concept

A user of the DTN-enabled GEO relay will receive benefits beyond those that are achieved by a bent-pipe relay. The most significant advantage, in the case of optical space-toground links, is the user is isolated from any issues that might be affecting the trunkline. A user can continue to transmit data to the relay (assuming enough relay storage) despite any trunkline outages. If custody transfer of the data is in place between the relay and user, the user can free up its onboard storage at this point. In a scenario in which a user is attempting to send data to one or more other users, all of the users do not need to have a link simultaneously. The data is essentially "pre-positioned" onboard the relay and the destination users receive the data when they are able to connect.

A reference mission concept for the LCRD mission is illustrated in Figure 2. The GEO relay may have multiple active links simultaneously. The links may be to any combination of user platforms and ground stations. The multiple ground station links may become active due to either pre-determined schedules or may have been established on relatively short notice due to changing weather conditions. The onboard store and forward capabilities allow for a user-to-relay link to remain active independently of any ground station handover that may be occurring. It may even be possible, through the use of routing individual bundles, to have a user's data forwarded to other users or multiple ground locations - perhaps even directly to a science archive facility. As long as there remains the storage capacity onboard the relay, the destinations for the user data do not need to be connected contemporaneously with the user. This allows the transmitting user to maintain its own storage and link transmission times, without consideration of any link schedule considerations past its own links to the relay.

The same benefits apply in the forward link direction, as well. User forward link data may be transmitted up to the relay before a user is connected, as long as there is the available trunkline bandwidth and onboard storage. Prepositioning forward link data in this way provides some isolation from any trunkline outages in the forward link direction. In some operational concepts, the forward link data may be for multiple users. The data can remain onboard the GEO relay until each destination becomes available.

In order to provide all of this flexibility for users, the relay network's mission operations center must assume the responsibility for the user data flows. This responsibility goes beyond establishing links based on a schedule. The MOC must now keep an accounting of the user data in transit within the provider system (onboard the relay or within a ground station). Any handovers or outages that require retransmissions or rerouting within the provider network must all be managed by the MOC transparently to the users. The MOC must also be able to provide the necessary insight to resolve any lost data issues reported by users. This increase of networking management complexity and responsibility is a significant change from the current NASA Space Network operations.

\section{SUMMARY/CONCLUSION}

The LCRD mission will provide a prototype for a future GEO relay network combining laser communications and space internetworking technologies. The two years of 
operations will allow the refinement and demonstrations of various operations concepts and applications, while also addressing the remaining challenges for the implementation of a future network It is considered to be a pathfinder for NASA putting a laser communications service on the Next Generation Tracking and Data Relay Satellite to be launched sometime next decade.

\section{REFERENCES}

[1] Bernard L. Edwards and Dave Israel, NASA Goddard Space Flight Center, Keith Wilson Jet Propulsion Laboratory, John Moores and Andrew Fletcher, MIT Lincoln Laboratory, "Overview of the Laser Communications Relay Demonstration Project" SpaceOps 2012

[2] K. E. Wilson, NASA Jet Propulsion Laboratory, California Institute of Technology and J. Moores, Massachusetts Institute of Technology Lincoln Laboratory, "The Architecture of the Laser Communications Relay Demonstration Ground Stations, an Overview", SPIE Photonics West 2013

[3] Jason Stewart, Dan Murphy, John Moores, Andrew Fletcher, Keith Bonneau, Massachusetts Institute of Technology Lincoln Laboratory, "Comparing adaptive optics approaches for NASA LCRD Ground Station \#2”, SPIE Photonics West 2013

[4] V. Cerf et al., Delay-Tolerant Network Architecture, IETF RFC 4838, informational, April 2007.

[5] "Operations Concept for a Solar System Internetwork (SSI)", Interagency Operations Advisory Group Report, October 2010.

[6] David J. Israel, Faith Davis, Jane Marquart, "A DTNBased Multiple Access Fast Forward Service for the NASA Space Network," smc-it, pp. 61-65, 2011 IEEE Fourth International Conference on Space Mission Challenges for Information Technology, 2011
Biographies

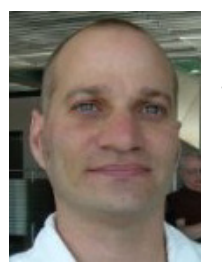

David J. Israel is the Principal Investigator for the Laser Communications Relay Demonstration (LCRD) and the Space Communications Manager in the Exploration and Space Communications Projects Division at Goddard Space Flight Center. He has been working on various aspects of space communications systems, since joining NASA in 1989. He received a B.S.E.E from Johns Hopkins University in 1989 and M.S.E.E. from George Washington University in 1996. He has led the development of various Space Network/Tracking and Data Relay Satellite System (TDRSS) operational systems and has been the principal investigator for multiple communications technology activities concerning advanced transceiver concepts and IP protocols, including the LPT CANDOS experiment on STS107. He was a member of the Interagency Operations Advisory Group (IOAG) Space Internetworking Strategy Group (SISG) and the lead for NASA Space Communications and Navigation Program Space Internetworking Study.

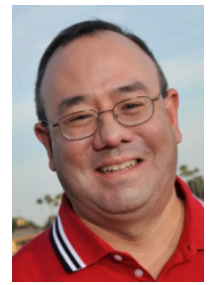

Bernard L. Edwards is the Chief Communications Systems Engineer at NASA GSFC. He received a B.S. in Electrical Engineering in 1989, a M.S. in Electrical Engineering in 1991, and a M.S. in Computer Science in 1993 all from the Johns Hopkins University. He is a member of the Interagency Operations Advisory Group (IOAG) Optical Link Study Group and helps represent NASA in the Consultative Committee for Space Data Systems (CCSDS) in the area of laser communications. He has been involved in various NASA laser communications projects and technology developments since coming to NASA in 2000.

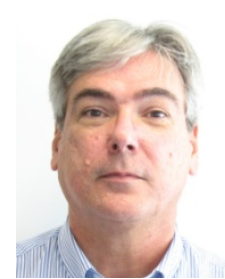

Donald E. Whiteman received a B.S. in Electrical Engineering from The University of Arizona in 1984 and a M.S. in Electrical Engineering from New Mexico State University in 1992. He has been at NASA GSFC since 2005 and is currently the LCRD Mission Systems Engineer. Prior to LCRD, he supported the NASA Space Communications and Navigation Program study efforts to develop a future communications and networking architecture for NASA. He has previously lead system engineering team efforts related to flight research, communications and instrumentation development at NASA DFRC. His career started in $R F$ and instrumentation system development at the Naval Air Warfare Center 